\title{
BLOCKADE OF RETROGRADE AXONAL TRANSPORT DELAYS THE ONSET OF METABOLIC AND MORPHOLOGIC CHANGES INDUCED BY AXOTOMY ${ }^{1}$
}

\author{
PHILIP A. SINGER, ${ }^{*}{ }^{\prime}{ }^{2}$ SHARON MEHLER, \\ Departments of * Neurology and \$Physiology, University of Kansas Medical Center, Kansas City, Kansas 66102 and \\ \$ Medical Research Service, Veterans Administration Medical Center, Kansas City, Missouri 64128
}

Received January 7, 1982; Revised April 14, 1982; Accepted April 21, 1982

\begin{abstract}
Axotomy-induced increase in 2-deoxyglucose (2-DG) uptake by motor nuclei and neuronal chromatolytic changes were studied after subepineural injection of colchicine into the motor nerve. Hypoglossal nuclei of either cats or rats were axotomized bilaterally, while one of the nerves was injected with colchicine or saline proximal to the site of nerve transection and the other was left intact or injected with saline. Colchicine abolished or decreased the uptake of 2-DG by axotomized nuclei and delayed the onset of chromatolysis. The decrease in 2-DG uptake was observed in rat hypoglossal nuclei between 24 and $48 \mathrm{hr}$ but not 5 days after drug treatment. In turn, a delay in the onset of chromatolysis was observed in cat hypoglossal nuclei at 14 days but not 30 days after treatment. Saline did not prevent chromatolysis nor the increased uptake of 2-DG. Colchicine injected intraneurally in intact preparations did not result in chromatolysis or in increased 2-DG uptake. Following colchicine injection, the drug remained localized near the site of injection and blocked retrograde axonal transport of horseradish peroxidase in the hypoglossal nerve. These findings suggest that the onset of chromatolysis and of the increase in 2-DG uptake after axotomy are partly dependent upon retrograde axonal transport.
\end{abstract}

When motor axons are injured, their cell bodies undergo a series of metabolic and morphologic changes which generally have been viewed as specifically conducive to regeneration (Grafstein, 1975). Among other changes, previous authors have shown that axotomized motor neurons show typical chromatolytic alterations in their morphology (Cammermeyer, 1969), increases in RNA synthesis (Watson, 1965), and profound modifications in protein metabolism (Brattgard et al., 1957, 1958). In addition, a recently described significant increase in 2deoxyglucose (2-DG) uptake by motor nuclei has been shown to be one of the first consequences of axotomy, even preceding the aforementioned changes (Kreutzberg and Emmert, 1980; Singer and Mehler, 1980). The factors involved in such an enhanced utilization of 2-DG are not known, but they may include, among other possibilities, an increase in the synthetic activity of neurons or in the sodium pump performance resulting from changes in

\footnotetext{
' This work was supported by the Veterans Administration Medical Research Service. Thanks are due to Dr. J. Espinosa for his involvement in the early stages of this work.

${ }^{2}$ To whom correspondence should be addressed at Neurology Service, Veterans Administration Medical Center, 4801 Linwood Boulevard, Kansas City, MO 64128.
}

membrane permeability. Whatever the case may be, since an increase in 2-DG uptake occurs as early as $24 \mathrm{hr}$ after axotomy, it may serve as a sensitive and timely marker to study the metabolic events initiated by axotomy.

The mechanisms underlying the initiation of the motor neuron's response to axonal injury are not fully understood, but several possible "triggering" signals have been proposed by other workers: depolarization of the membrane, loss of action potentials, loss of axoplasm, lack of substances conveyed by retrograde axonal flow, and/or a substance originating at the site of injury and conveyed to the cell body by retrograde axonal transport (Cragg, 1970; Grafstein, 1975). Previous experiments by others have shown that application of botulinum toxin to the nerve terminals, a treatment which does not cause any significant ultrastructural changes (Thesleff, 1960), results in alterations in nucleic acid metabolism like those seen during regeneration (Watson, 1969). These previous experiments imply that neither axonal membrane changes nor a possible loss of axoplasm is absolutely essential as signals for the initiation of the regeneralive response. In turn, the dependence of the time of onset of regenerative changes on the distance between axonal lesions and the cell body suggests that retrograde axonal 
transport might be involved in supplying some molecular signal to the cell body (Cragg, 1970). Although a role for retrograde axonal transport has been suggested previously, there is no direct evidence to support such a hypothesis.

To study further the possible involvement of axonal transport in the onset of motor neuron regenerative events, we have examined the effects of transport interruption on certain indicators of regeneration in axotomized neurons. Specifically, we have studied the onset of chromatolytic changes and the increase in 2-DG uptake induced by nerve injury. Preliminary accounts of some of our results have been reported elsewhere (Espinosa and Fernandez, 1976; Fernandez et al., 1981).

\section{Materials and Methods}

Methods. Adult male cats $(2.5$ to $4.0 \mathrm{~kg}$ ) or male Sprague-Dawley rats (120 to $150 \mathrm{gm})$ were anesthetized with sodium pentobarbital $(50 \mathrm{mg} / \mathrm{kg})$ administered intraperitoneally. The left hypoglossal nerve was injected locally with either $5 \mu \mathrm{l}$ of $10 \mathrm{~mm}$ colchicine (experimental) or $5 \mu \mathrm{l}$ of saline (control) under the epineurium close to the nerve's bifurcation near the carotid artery. The injection was made through a 33 gauge needle with the aid of a micromanipulator and dissecting microscope (Fernandez and Ramirez, 1974). Sufficient bromphenol blue was added to the solutions to monitor the progress of the injections visually.

Subsequently, in most animals, both branches of the injected and the contralateral intact nerves were transected $5 \mathrm{~mm}$ distal to the injection site and the animals were allowed to recover. Four rats were studied at 5 days and another four at 10 days after nerve section. Because of the difficulty that they experienced eating with a totally denervated tongue, only the left hypoglossal nerve was injected and sectioned in these animals. In two rats, the nerves were left intact after the colchicine injections, and in some cats, one nerve was injected with colchicine and the other side was injected with saline. In some of these cats, the nerves were left intact after colchicine or saline injection, and in others, they were transected as above. In some experiments on rats, ${ }^{3} \mathrm{H}$-labeled colchicine was injected into the intact nerve by means of the aforementioned techniques, and on other occasions, the unlabeled drug $(10 \mu l)$ or an equal quantity of methylene blue was injected directly into the floor of the fourth ventricle. In the former instance, the atlanto-occipital membrane was exposed under sodium pentobarbital anesthesia and the dura was removed. Following injection, the cervical muscles were approximated, the wound was closed, and the animal was allowed to recover. After 24 $\mathrm{hr}$, the colchicine-injected animals were prepared for 2DG analysis. Additional experiments on rats involved local bilateral injections ( $10 \mu \mathrm{l}$ each) of $10 \%$ horseradish peroxidase (HRP) into the tongue muscles and injection of colchicine into one of the hypoglossal nerves.

In all of the experiments at time intervals between 1 $\mathrm{hr}$ and 60 days, the animals were either prepared for 2DG analysis or reanesthetized with $50 \mathrm{mg} / \mathrm{kg}$ of sodium pentobarbital (i.p.) and the brainstem was removed for morphologic analysis. In the animals injected intraneurally with $\left[{ }^{3} \mathrm{H}\right]$ colchicine, the entire hypoglossal nerve and the geniohyoid, genioglossus, and hyoglossus muscles also were removed.

2-Deoxyglucose. Twenty-four, 36 , and $48 \mathrm{hr}$ and 5 and 10 days after the injection and nerve transection, $15 \mu \mathrm{Ci}$ / $100 \mathrm{gm}$ of $\left[{ }^{14} \mathrm{C}\right] 2-\mathrm{DG}(51.3 \mathrm{mCi} / \mathrm{mmol})$ was injected into the awake rats intravenously via the tail vein. Forty-five min later, the animals again were anesthetized with sodium pentobarbital $(50 \mathrm{mg} / \mathrm{kg}$, i.p.) and the brainstem was exposed, removed, mounted on a cork, and frozen in Freon cooled to $-70^{\circ} \mathrm{C}$. The blocks were stored at $-70^{\circ} \mathrm{C}$ until sectioning. At the time of sectioning, the blocks were allowed to equilibrate to $-18^{\circ} \mathrm{C}$ overnight in a cryostat. Coronal sections ( $20 \mu \mathrm{m}$ thick) were cut, placed on glass slides, dried, and then applied to Kodak MINAR $\mathrm{x}$-ray film for 2 weeks at room temperature. Enlarged transparencies of the resultant autoradiographs were made and the optical density of the two hypoglossal nuclei was compared using a Tobius TBX densitometer. The differences were compared using the paired $t$ test. Sections from each animal were stained with toluidine blue to verify the location of the hypoglossal nuclei.

$\left[{ }^{3} \mathrm{H}\right]$ Colchicine. The $\left[{ }^{3} \mathrm{H}\right]$ colchicine was injected into the nerve as noted above $(1 \mu \mathrm{Ci} / 5 \mu \mathrm{l}$ of saline; $5 \mathrm{Ci}$ / $\mathrm{mmol}$ ), and the distribution of the drug was studied from 1 to $24 \mathrm{hr}$ after injection. Serial sections $(2 \mathrm{~mm}$ ) of nerve and samples of muscle, as well as regions of the floor of the fourth ventricle containing hypoglossal nuclei, were digested in $300 \mu \mathrm{l}$ of NCS tissue solubilizer and the radioactivity was assessed by liquid scintillation counting (Inestrosa and Fernandez, 1976).

Morphology. Cats were anesthetized with sodium pentobarbital $(50 \mathrm{mg} / \mathrm{kg}$, i.p.) and perfused through each of the carotid arteries with $100 \mathrm{ml}$ of normal saline followed by $10 \%$ formalin. The brainstem was removed and fixed overnight in $10 \%$ formalin; the specimen then was trimmed, washed, dehydrated in alcohols, and embedded in paraffin. Horizontal sections $(7 \mu \mathrm{m})$ were cut and stained with Nissl stain. On a particular section, the entire nucleus was surveyed for morphologic changes and representative samples were chosen.

Methylene blue. The brainstems of rats injected with methylene blue into the floor of the fourth ventricle were removed $10 \mathrm{~min}$ after the injection and frozen on a cork in Freon at $-70^{\circ} \mathrm{C}$. They were allowed to equilibrate to $-18^{\circ} \mathrm{C}$ in a cryostat, $20-\mu \mathrm{m}$ sections were cut, and the sections were mounted on slides for viewing and photography.

Horseradish peroxidase. Twenty-four hours after the injection of HRP into the tongue, the animals were reanesthetized and perfused through the heart with 50 $\mathrm{ml}$ of saline followed by $200 \mathrm{ml}$ of phosphate-buffered $1.25 \%$ glutaraldehyde, $1 \%$ paraformaldehyde. The brainstem was removed and placed in the buffered glutaraldehyde/paraformaldehyde solution overnight at $4^{\circ} \mathrm{C}$. At the time of sectioning, the brainstem was mounted on a cork and frozen in Freon cooled to $-70^{\circ} \mathrm{C}$. The blocks were allowed to warm to $-18^{\circ} \mathrm{C}$ in the cryostat, and 20 $\mu \mathrm{m}$ sections cut and free-floated in phosphate-buffered sucrose/ethylene glycol solution. They then were washed in three changes of phosphate-buffered sucrose/ethylene glycol. The sections were subsequently incubated in acetate-buffered sodium nitroprusside with tetramethylben- 

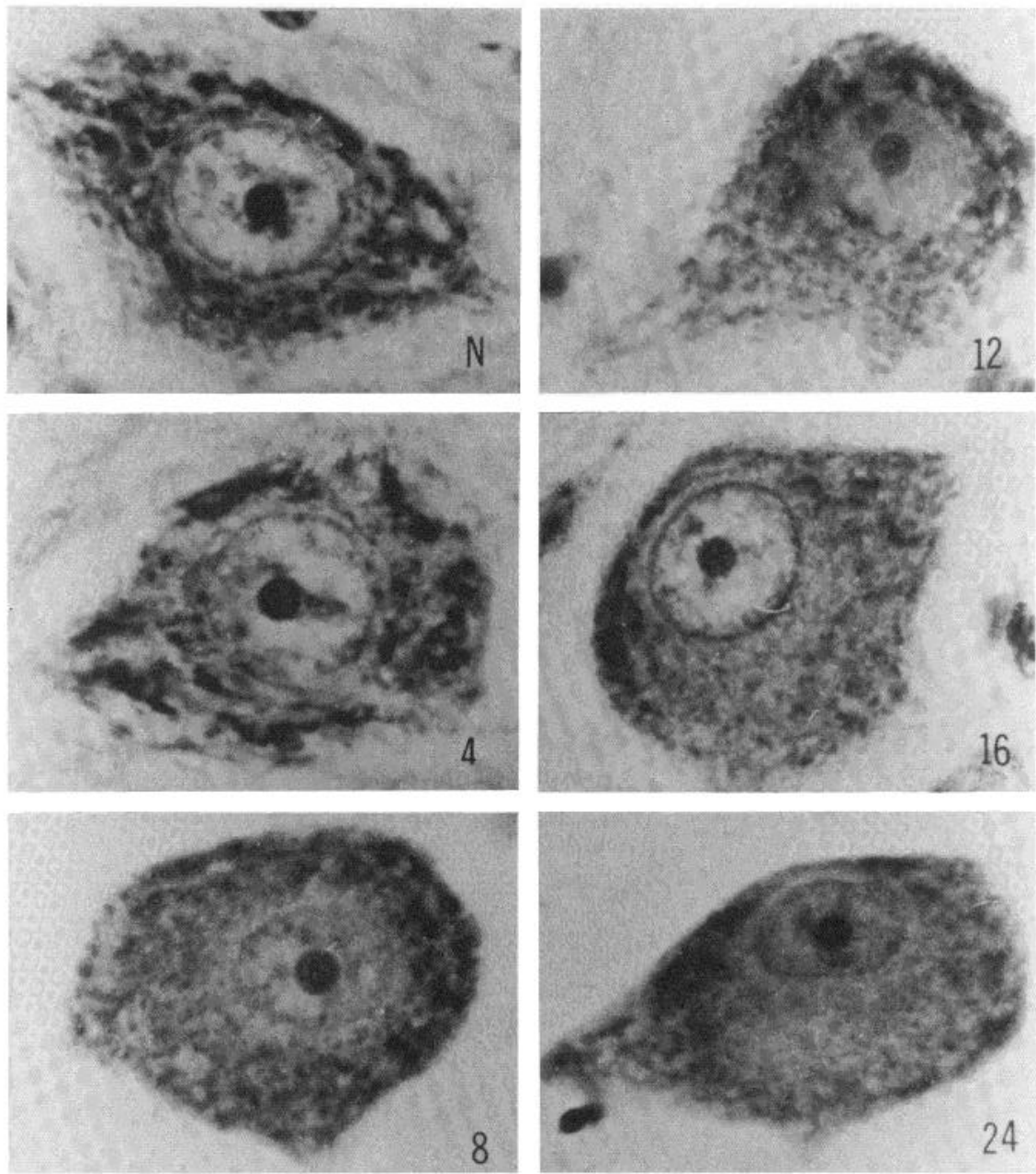

Figure 1. Representative photomicrographs of cat hypoglossal neurons at different times $(4,8,12,16$, and 24 days) after transection of one hypoglossal nerve ( $5 \mathrm{~mm}$ distal to its bifurcation). The contralateral nerve was left intact so that the corresponding neurons served as a control $(N)$. Paraffin sections $(7 \mu \mathrm{m})$ were stained with Nissl stain. Overall magnification $\times 2000$.

zene for $7 \mathrm{~min}$ and transferred to fresh sucrose/ethylene glycol solution, with hydrogen peroxide, for an additional 7 -min period. The sections were washed in sucrose/ethylene glycol solution, placed on slides, air-dried, and mounted with Permount.

Materials. $\left[{ }^{14} \mathrm{C}\right] 2$-Deoxyglucose, $\left[{ }^{3} \mathrm{H}\right]$ colchicine, and NCS solubilizer were obtained from New England Nuclear Corp.; horseradish peroxidase, histological stains, and reagents were all obtained from Sigma.

\section{Results}

The effects of hypoglossal axotomy in the cat were studied $4,8,12,16,24,30$, and 40 days after nerve transection. Slight chromatolytic changes could be observed at 4 and 8 days, but the morphologic changes became most apparent after 12 days. Typical signs of chromatolysis included swelling of the cell bodies, dispersion of the Nissl substance, and displacement of the nucleus toward the periphery of the cell soma (Fig. 1). Similar changes were seen in preparations in which the solvent was injected into the hypoglossal nerve and axotomy was performed distal to the injection 14 days prior to analysis. By contrast, when colchicine, instead of solvent, was injected into the contralateral nerve and distal axotomy was performed, no clear signs of chromatolysis were detected after 14 days of distal axotomy (Fig. 2). Under these conditions, however, chromatolysis was observed after 30 and 40 days. Colchicine injected either stereotaxically within the hypoglossal nucleus or into the intact hypoglossal nerve produced no chromatolytic changes in the same time period. In the latter case, the results of colchicine injection were compared with those of solvent injection into the contralateral nerve and no difference was observed (Fig. 3 ). In addition, the results from both colchicine and solvent injections were indistinguishable from normal.

$\left[{ }^{14} C\right] 2-D G$ and intraneural colchicine. There was a marked increase in 2-DG uptake from $24 \mathrm{hr}$ to 10 days in all hypoglossal nuclei whose nerves were axotomized (Figs. 4 and 5) without colchicine injection. This was also the case in nuclei whose nerves were cut and injected with saline proximal to the cut (Fig. $4 B$ ). In animals injected in one nerve with colchicine and with no manipulation on the contralaleral side, there was no increased 

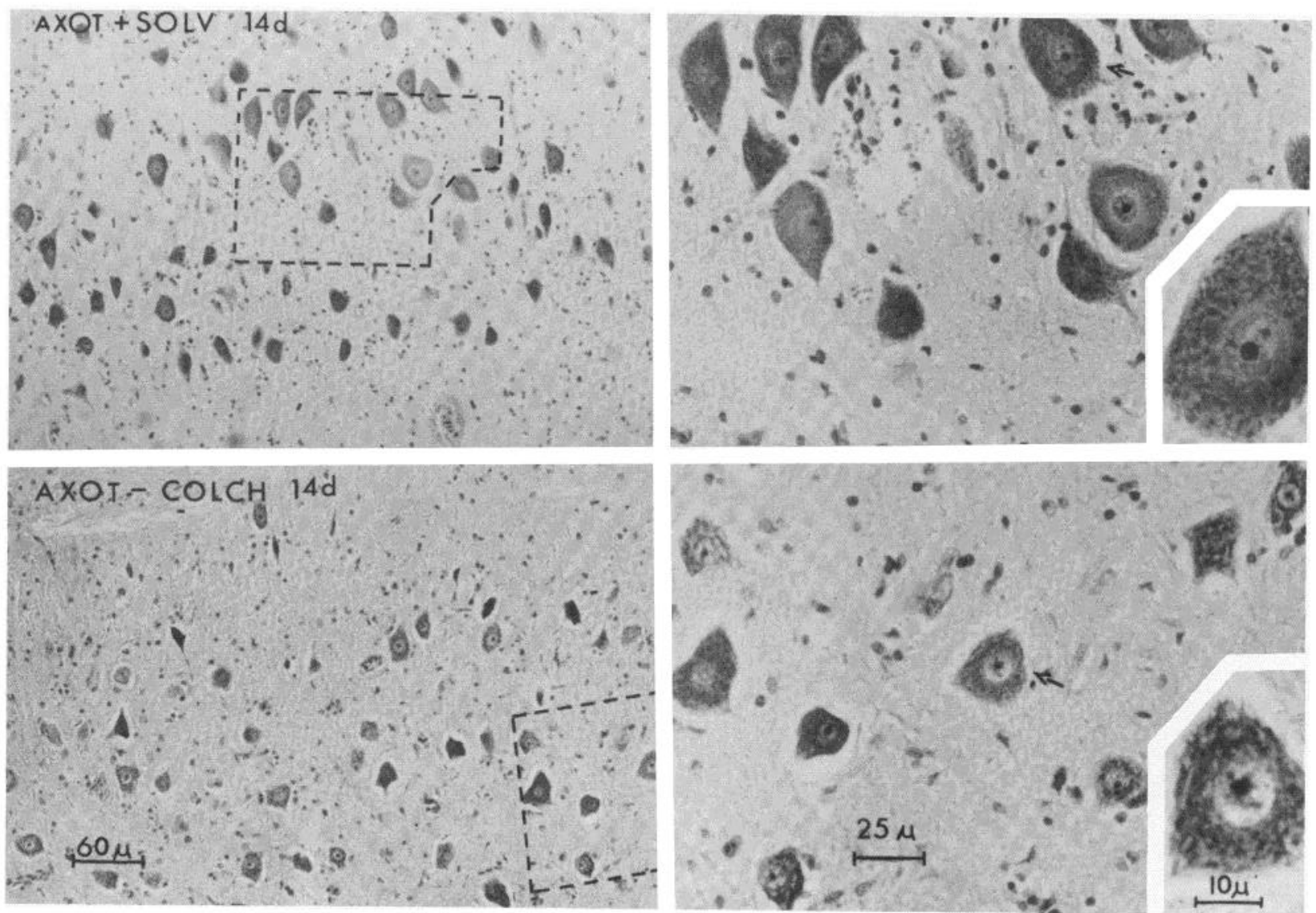

Figure 2. Photomicrographs of horizontal sections through cat hypoglossal nuclei. The right hypoglossal nerve was transected and injected with $10 \mathrm{~mm}$ colchicine $(5 \mu \mathrm{l})$ proximal to the cut (bottom), whereas the left hypoglossal nerve was transected and injected with solvent (top). After 14 days, the brainstem was fixed, embedded in paraffin, sectioned (7- $\mu \mathrm{m}$ sections), and stained with Nissl stain. The dashed areas on the left photographs correspond to the areas shown on right photographs. The arrows indicate the neurons illustrated by the insets.

2-DG uptake on either side (Fig. $4 C$ ). However, in four of nine animals at $24 \mathrm{hr}$ (Fig. $4 A$ ), one of three at $36 \mathrm{hr}$ (Fig. $5 A$ ), and one of two at $48 \mathrm{hr}$ (Fig. $5 B$ ), there was no 2-DG uptake above background on the side of the transection and colchicine injection. In the other five animals at $24 \mathrm{hr}$, one at $36 \mathrm{hr}$, and one at $48 \mathrm{hr}$, there was some increase in 2-DG uptake on the side of the axotomy and colchicine injection but less than on the side of axotomy alone. In one animal at $36 \mathrm{hr}$, there was no difference between the two sides. Optical density analysis of the autoradiograms at $24 \mathrm{hr}$ showed a significant ( $p=0.0008$ ) difference between the axotomized side injected with colchicine and the side of axotomy alone (Table I). There was a slightly greater density on the side of the axotomy and saline injection than on the side of the axotomy alone. There was also a very slight difference between the results obtained from the colchicine alone side and the side receiving no manipulation (Table I).

The animals whose nerves were cut and injected with colchicine 5 and 10 days before sacrifice showed increased uptake of 2-DG on the axotomized side (Fig. $5 C$ ). Although this was done only on one side while the contralateral nerve was left intact, the intensity of the uptake appeared the same as in axotomized noninjected animals. Colchicine (10 $\mu \mathrm{l}, 20 \mathrm{~mm}$ in saline) was injected directly into the brainstem in two rats. No increase was seen in the nuclei, although there was increased 2-DG uptake as well as an inflammatory response at the site of the injection. Under the above conditions, colchicine probably reached the hypoglossal nucleus since, when methylene blue $(10 \mu \mathrm{l})$ was injected in the same manner in two other rats, the neurons of the hypoglossal nucleus were clearly stained.

Distribution of $\left[{ }^{3} \mathrm{H}\right]$ colchicine in the nerve. The distribution of $\left[{ }^{3} \mathrm{H}\right]$ colchicine along the hypoglossal nerve was studied from 1 to $24 \mathrm{hr}$ after the injection. Within the first few minutes, the only radioactivity detected in the whole nerve was that localized at the site of injection. Over the following hours ( 1 to $6 \mathrm{hr}$ ), the radioactivity in the injection site gradually decreased as it diffused in both directions along the nerve. The total amount of radioactive label decreased with time, and after $6 \mathrm{hr}$, no counts above background level could be found along the entire nerve (Fig. 6). Throughout the testing period, no significant amounts of radioactivity were detected in: $(a)$ the nerve trunk beyond 1 to $1.5 \mathrm{~cm}$ either proximal or distal to the injection site; $(b)$ the geniohyoid, genioglossus, and hyoglossus muscles; and $(c)$ the region of the medulla oblongata containing the hypoglossal nuclei. These results confirm previous findings (Fernandez and 

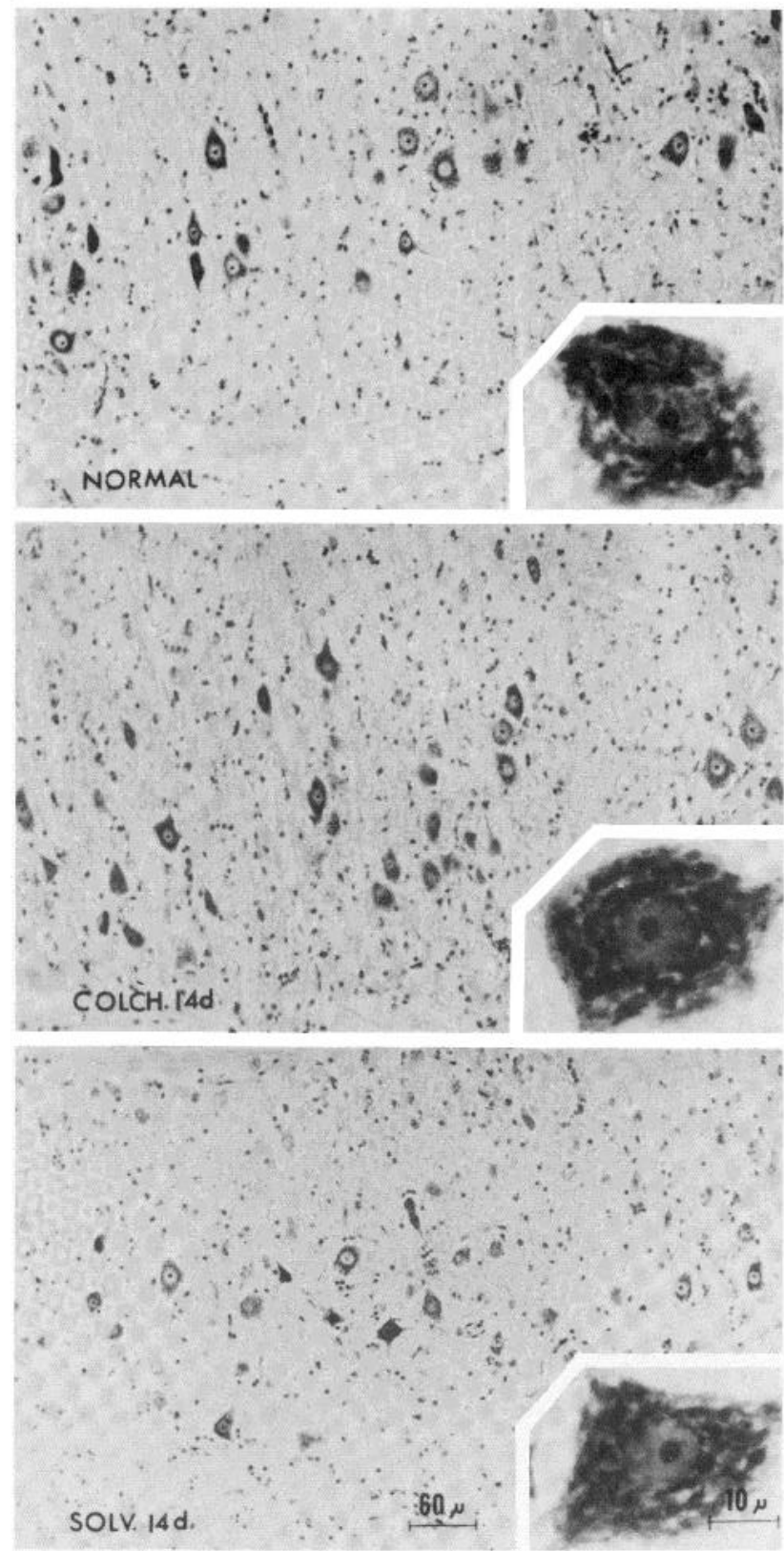

Figure 3. Photomicrographs of horizontal sections through cat hypoglossal nuclei (top, normal) 14 days after either colchicine (middle) or solvent (bottom) injections into the hypoglossal nerve. Paraffin sections $(7 \mu \mathrm{m})$ were stained with Nissl stain. The insets show enlargements of representative neurons in the three conditions.

Ramirez, 1974; Inestrosa and Fernandez, 1976) as to the temporary persistence of colchicine in the area of local injection.

Horseradish peroxidase. Three of the four animals whose tongues were injected bilaterally with HRP had staining of the hypoglossal cell bodies $24 \mathrm{hr}$ later on the intact side. On the side in which colchicine was injected into the nerve trunk, there was no staining of the hypoglossal neurons (Fig. 7). In one animal, no staining of hypoglossal neurons on either side occurred.

\section{Discussion}

These experiments show that colchicine injection proximal to the site of axotomy delayed the increase in 2-DG uptake as well as the onset of chromatolysis. The morphologic changes were definite although difficult to quantitate. Since the more easily measured increase in 2-DG uptake has been shown to be one of the earliest changes after axotomy (Singer and Mehler, 1980), we chose this parameter as an indicator of regeneration. However, the mechanism of the process producing this increased glucose use is unknown.

Since colchicine blocks retrograde axoplasmic flow, our experiments do clarify some aspects of the increased 2DG uptake after axotomy. After $24 \mathrm{hr}$, following uptake by nerve terminals and retrograde axonal transport (Kristensson et al., 1971), horseradish peroxidase was detected only in neuronal cell bodies of hypoglossal fibers not treated with colchicine. This finding is in agreement with previous studies showing that colchicine effectively blocks retrograde axonal transport (Kristensson and Sjostrand, 1972) without altering impulse conduction (Hinkley and Green, 1971; Perisic and Cuenod, 1972). The prevention of 2-DG uptake by colchicine suggests that, after nerve transection, the early increase in 2-DG utilization is dependent on retrograde axonal transport rather than electrical membrane activity or altered membrane potential originating at the site of axotomy.

Thus far, the 2-DG technique has not permitted a resolution adequate to determine whether the changes observed actually occur within the neuronal cell bodies (Kreutzberg and Emmert, 1980; Singer and Mehler, 1980). Nonetheless, we have demonstrated that, under the conditions used, colchicine also prevents chromatolysis in the hypoglossal neurons. Accordingly, our results support the hypothesis that a substance(s) which triggers certain neuronal regenerative changes is either produced or altered at the site of nerve injury and reaches the soma by means of retrograde axonal transport. On the other hand, it is also possible that anterograde axonal transport alterations resulting from nerve injury could cause some depletion of protein in the cell body, thereby initiating the regenerative changes (Cragg, 1970). Since colchicine also blocks anterograde axoplasmic transport (Dahlstrom, 1968), it could have blocked such a signaling mechanism. It must be noted, however, that changes in anterograde axoplasmic transport have been reported to occur much later than the early 2-DG uptake changes reported here (Grafstein, 1975).

Other investigators have suggested that, in normal neurons, regenerative changes are suppressed by a substance carried from the periphery which is lost upon nerve transection (Kristensson and Sjostrand, 1972). Partial support for this possibility comes from experiments showing that regenerative changes also may occur after application of colchicine to chicken ciliary nerves (Pilar and Landmesser, 1972). In those experiments, however, the concentration of colchicine used was 50 -fold higher than that used by us and because of the small distance between the site of application and the cell bodies, the changes reported could have been caused in that prepa- 

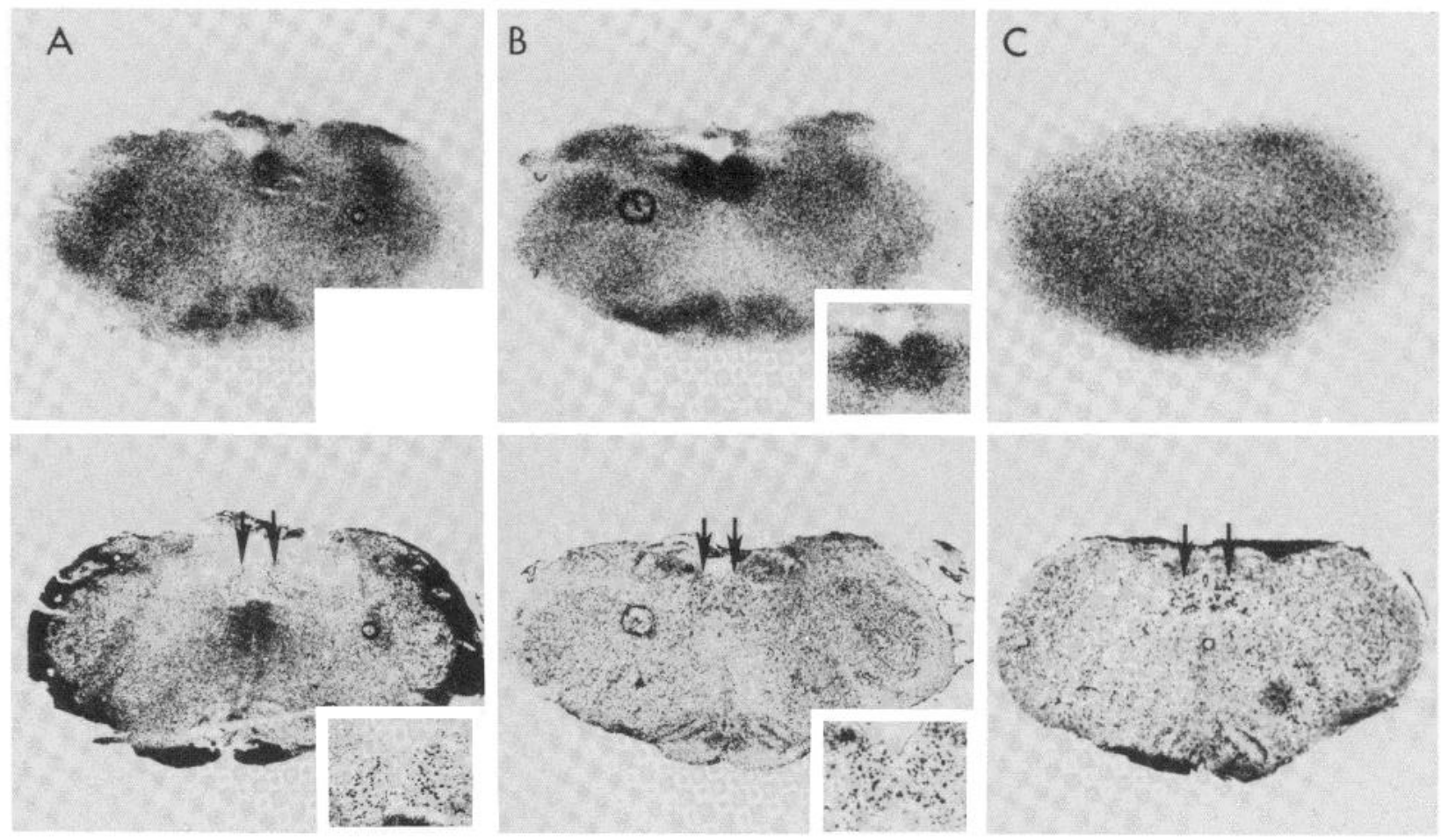

Figure 4. $\left[{ }^{14} \mathrm{C}\right] 2$-Deoxyglucose coronal autoradiographs (top) and corresponding micrographs (below) of rat hypoglossal nuclei. The inset shows an enlargement of the area of the hypoglossal nuclei. The arrows indicate the hypoglossal nuclei. $A, 24 \mathrm{hr}$ after bilateral axotomy with colchicine injected into the left hypoglossal nerve proximal to the axotomy; $B, 24 \mathrm{hr}$ after bilateral axotomy with saline injected proximal to the axotomy site on the left; $C, 24 \mathrm{hr}$ after colchicine injection into the left hypoglossal nerve.
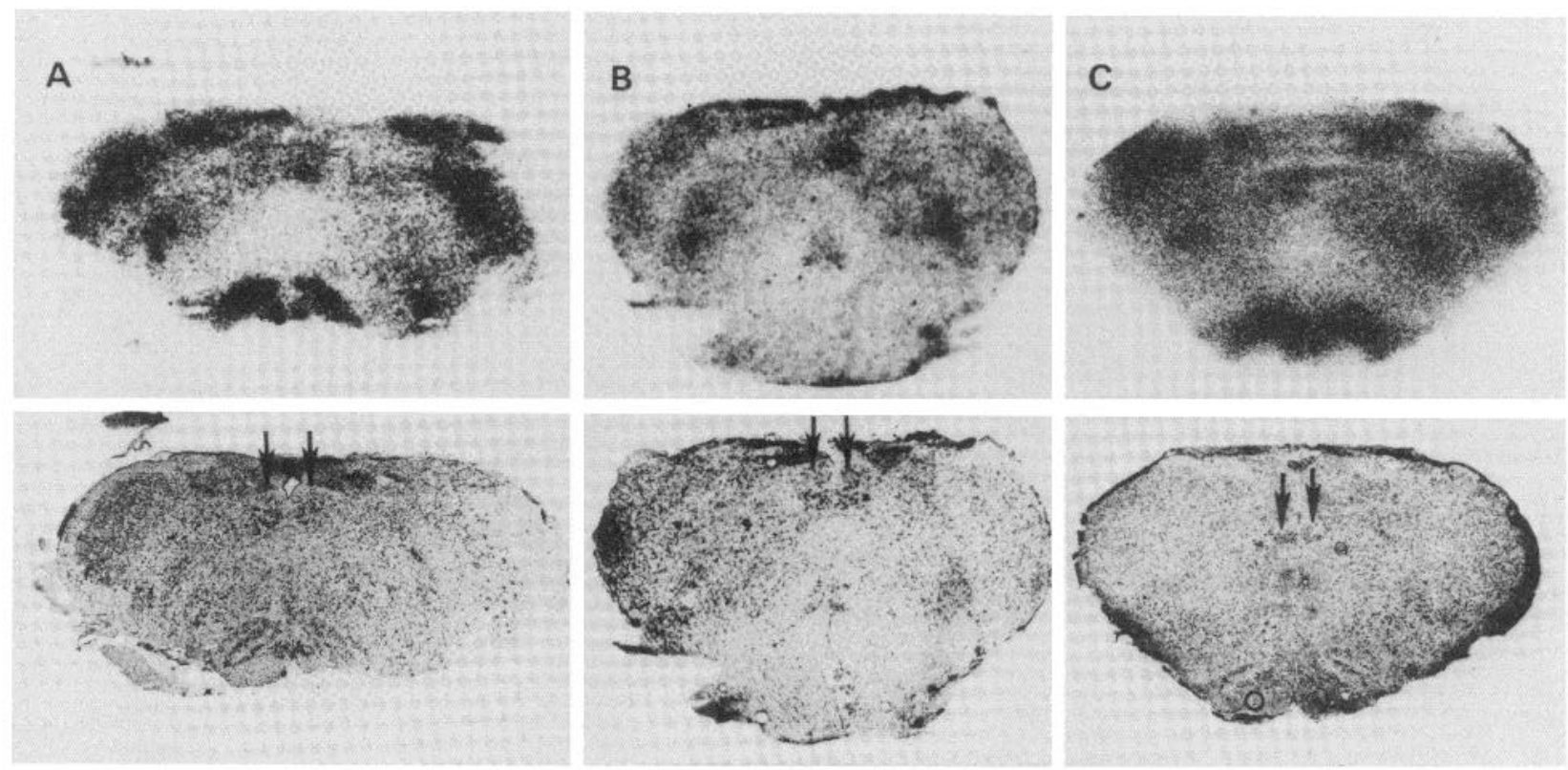

Figure 5. $\left[{ }^{14} \mathrm{C}\right] 2$-Deoxyglucose coronal autoradiographs (top) with corresponding micrographs ( below) of rat hypoglossal nuclei. The arrows indicate the hypoglossal nuclei. $A, 36 \mathrm{hr}$ and, $B, 48 \mathrm{hr}$ after bilateral axotomy with colchicine injected intraneurally proximal to the axotomy on the left. $C, 5$ days after axotomy on the left only with proximal intraneural colchicine injection.

ration by the drug acting directly on the neuronal cell bodies. In the present case, colchicine, whether injected into the hypoglossal nerve or into the brainstem, did not produce regenerative changes but blocked their initiation. Accordingly, we conclude that, whatever the nature and mechanisms of action of the signal that initiates regenerative changes, it is mediated by retrograde axoplasmic transport. Although a role for retrograde axoplasmic transport in initiating regeneration has been proposed previously by other authors (Cragg, 1970; Grafstein, 1975), ours is the first experimental data to support this suggestion directly. 
TABLE I

Optical density measurements of the hypoglossal nuclei

In the experimental group, a bilateral axotomy was performed and colchicine was injected proximal to the axotomy on the left. Control 1 includes those animals which received a bilateral axotomy with saline injected proximal to the axotomy on the left. Control 2 represents those on which no axotomy was performed but in which colchicine was injected into the left hypoglossal nerve.

\begin{tabular}{lccc}
\hline \multicolumn{1}{c}{ Animal } & O.D.L. $^{a}$ & O.D.R. & O.D.R. - O.D.L. \\
\hline Experimental & & & \\
262 & 0.78 & 0.93 & 0.15 \\
263 & 0.43 & 0.55 & 0.12 \\
265 & 0.45 & 0.52 & 0.07 \\
266 & 0.38 & 0.44 & 0.06 \\
281 & 0.34 & 0.46 & 0.12 \\
283 & 1.23 & 1.55 & 0.32 \\
284 & 0.34 & 0.50 & 0.16 \\
325 & 0.81 & 0.99 & 0.18 \\
327 & 0.75 & 0.81 & 0.06 \\
& & & $\bar{x}=0.14 \pm 0.08$ \\
& & & $p=0.0008$ \\
Control 1 & & & -0.02 \\
287 & 1.03 & 1.01 & -0.02 \\
288 & 1.20 & 1.18 & $\bar{x}=0.02$ \\
& & & \\
Control 2 & & & -0.01 \\
313 & & & -0.02 \\
314 & 0.31 & 0.30 & -0.015 \\
& 0.29 & 0.27 & \\
\hline
\end{tabular}

${ }^{a}$ O.D., optical density; L., left hypoglossal nerve; R., right hypoglossal nerve.
Regarding the time course for the action of colchicine, there appeared to be an increased 2-DG uptake 5 days after the injection of colchicine proximal to axotomy even though a decrease was seen in some animals at 36 and $48 \mathrm{hr}$ after injection. The morphologic experiments demonstrated inhibition of chromatolysis 14 days after injection but not at 30 and 40 days. The effect of colchi-

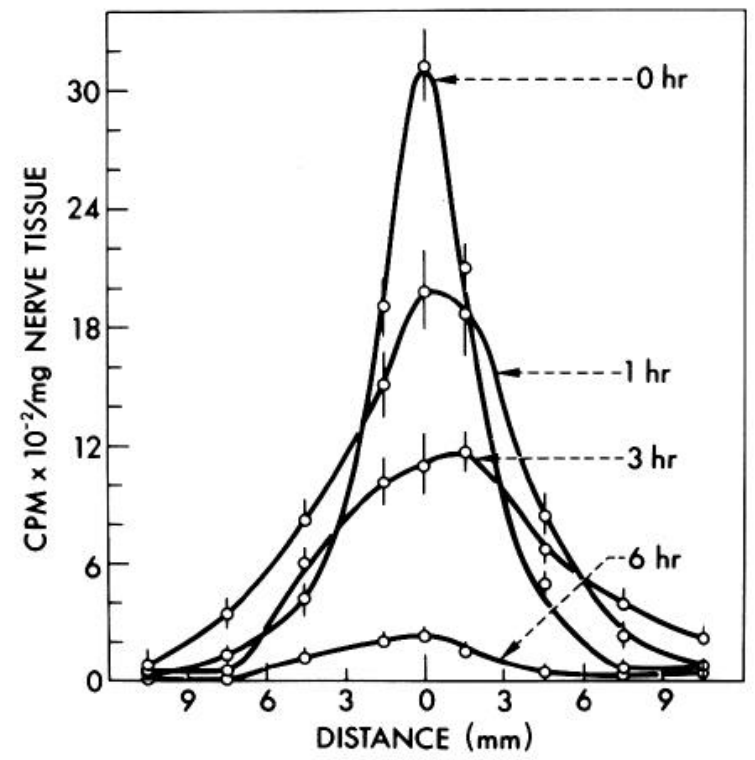

Figure 6. Distribution of $\left[{ }^{3} \mathrm{H}\right]$ colchicine along the rat hypoglossal nerve. Radioactivity (counts per $\mathrm{min} \times 10^{-2}$ per $\mathrm{mg}$ of nerve tissue) was measured along the nerve (1-mm segments) at different times after local injection $(0 \mathrm{~mm}$, site of injection) of the drug $(1 \mathrm{mCi} / 5 \mu \mathrm{l}$ in saline $)$. Each point represents the average $\pm \mathrm{SE}$ of four experiments.

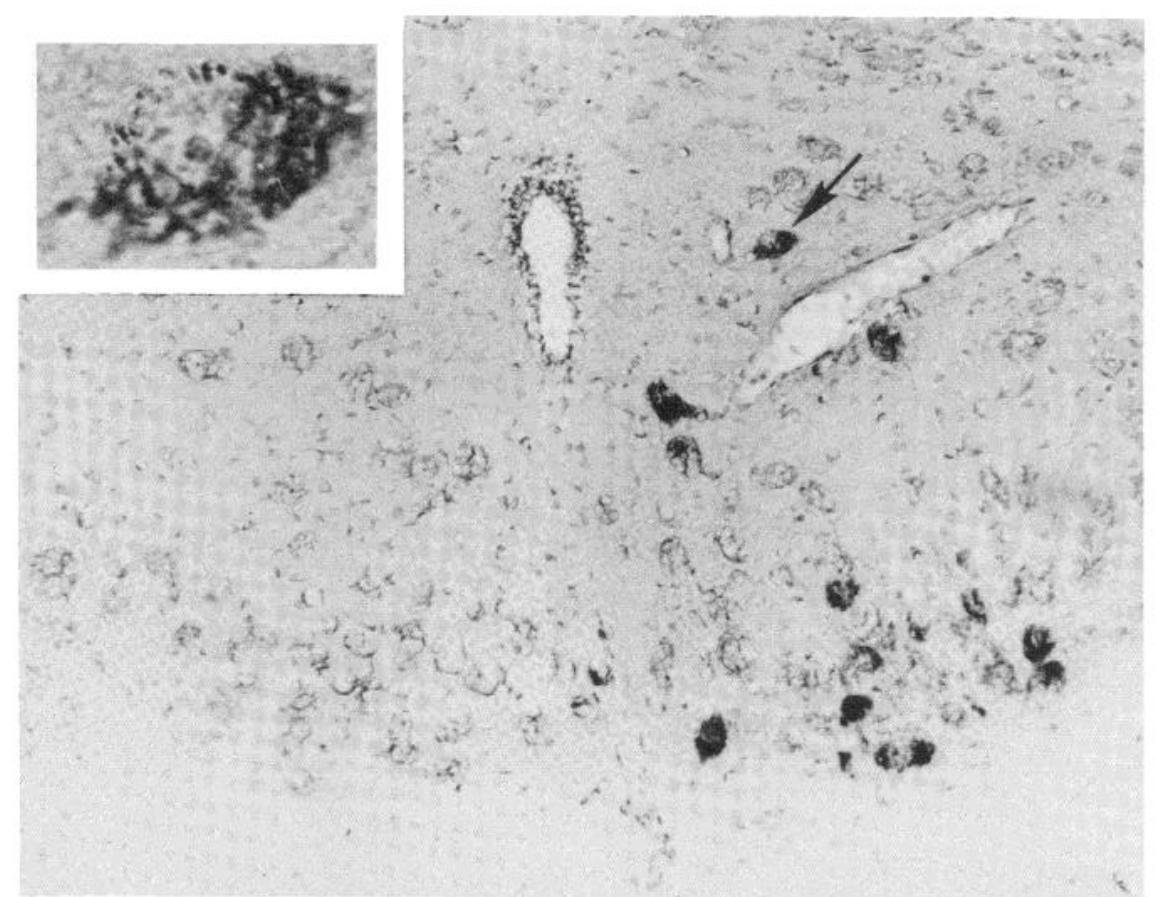

Figure 7. Photomicrograph of rat hypoglossal nuclei stained with horseradish peroxidase. Horseradish peroxidase was injected into both sides of the tongue $24 \mathrm{hr}$ previously. Colchicine was injected intraneurally into the left hypoglossal nerve at the level of the carotid artery. The right nerve was left intact. The inset is an enlargement of the area indicated by the arrow. Magnification $\times 100$. 
cine therefore is not permanent but rather regenerative events reappear after a temporary halt. This could be either because the drug becomes dilute and retrograde axonal transport is re-established or because some other mechanism becomes activated with time.

Since blockade of retrograde axoplasmic flow delays regenerative phenomena in the cell body, it is quite possible that substances liberated at the injury site ascend the axon and initiate regeneration. If this is the case, isolation of these substances could provide a mechanism for initiating or enhancing regenerative phenomena in the peripheral nervous system.

\section{References}

Brattgard, S. O., J. D. Edstrom, and H. Hyden (1957) The chemical changes in regenerating neurons. J. Neurochem. 1: 316-325.

Brattgard, S. O., H. Hyden, and H. Sjostrand (1958) Incorporation of orotic acid- ${ }^{14} \mathrm{C}$ and lysine $-{ }^{14} \mathrm{C}$ in regenerating single nerve cells. Nature 182: 801-802.

Cammermeyer, J. (1969) Species differences in acute retrograde neuronal reaction of the facial and hypoglossal nuclei. J. Hirnforsch. 11: 13-29.

Cragg, B. C. (1970) What is the signal for chromatolysis? Brain Res. 23: 1-21.

Dahlstrom, A. (1968) Effect of colchicine on transport of amine storage granules in sympathetic nerves of rats. Eur. J. Pharmacol. 5: 111-113.

Espinosa, J. J., and H. L. Fernandez (1976) Axoplasmic transport influence on neuronal chromatolysis. Arch. Biol. Med. Exp. (Santiago) 19: 156.

Fernandez, H. L., and B. U. Ramirez (1974) Muscle fibrillation induced by blockage of axoplasmic transport in motor nerves. Brain Res. 79: 385-395.
Fernandez, II. L., P. A. Singer, and S. Mehler (1981) Retrograde axonal transport mediates the onset of regenerative changes in the hypoglossal nucleus. Neurosci. Lett. 25: 7-11.

Grafstein, B. (1975) The nerve cell body response to axotomy. Exp. Neurol. 48: 32-51.

Hinkley, R. B., and L. S. Green (1971) The effects of halothane and colchicine on microtubules and electrical activity of rabbit vagus nerves. J. Neurobiol. 2: 97-106.

Inestrosa, N. C., and H. L. Fernandez (1976) Muscle enzymatic changes induced by blockage of axoplasmic transport. J. Neurophysiol. 39: 1239-1245.

Kreutzberg, G. W., and A. Emmert (1980) Glucose utilization of motor nuclei during regeneration: $A\left[{ }^{14} \mathrm{C}\right]$-2-deoxyglucose study. Exp. Neurol. 70: 712-716.

Kristensson, K., and J. Sjostrand (1972) Retrograde transport of protein tracer in rabbit hypoglossal nerve during regeneration. Brain Res. 45: 175-181.

Kristensson, K., I. Olsson, and J. Sjostrand (1971) Axonal uptake and retrograde transport of exogenous proteins in hypoglossal nerve. Brain Res. 32: 399-406.

Perisic, M., and M. Cuenod (1972) Synaptic transmission depressed by colchicine blockade of axoplasmic flow. Science 175: $1140-1149$

Pilar, G., and L. J. Landmesser (1972) Axotomy mimicked by localized colchicine application. Science 177: 1116-1118.

Singer, P., and S. Mehler (1980) 2-Deoxy $\left[{ }^{14} \mathrm{C}\right]$ glucose uptake in rat hypoglossal nucleus after nerve transection. Exp. Neurol. 69: $617-626$.

Thesleff, S. (1960) Super-sensitivity of skeletal muscle produced by botulinum toxin. J. Physiol. (Lond.) 151: 598-607.

Watson, W. E. (1965) An autoradiographic study of the incorporation of nucleic acid precursors by neurons and glia during nerve regeneration. J. Physiol. (Lond.) 180: 741-753.

Watson, W. E. (1969) The response of rat hypoglossal neurons to injection of botulinum toxin into the tongue. J. Physiol. (Lond.) 202: 101P. 